\title{
Utilise Higher Modulation Formats with Heterogeneous Mobile Networks Increases Wireless Channel Transmission
}

\author{
Heba Haboobi, Mohammad R. Kadhum \\ Faculty of Arts, Science \& Technology, University of Northampton, Northampton, \\ U.K. \\ heba.haboobi@northampton.ac.uk
}

\begin{abstract}
In this paper, a higher modulation format with a heterogeneous mobile network (small cells, Macrocells) is proposed, explored and evaluated at a wireless transmission system. As such, study the effect of utilising developed schemes of modulation like the 256 Quadrature Amplitude Modulation (QAM) on the modulation/de-modulation level of the currently applied Orthogonal Frequency Division Multiplexing (OFDM). Since the higher bit-rate of transmission is one of the important topics for the forthcoming generation of mobile, the introduced system aims to regulate the trade-off relationship between the maximum achieved bit-rate and the minimum required level of the Signal-to-Noise Ratio (SNR). Hence, involve the small cell technology as a supportive tool for the higher schemes of modulation to increase the capacity of the channel at the accepted limit of error. Consequently, the presented system that combines both the higher modulation formats and the small cells can expand the transmission coverage with a higher bit-rate yet keeping a similar level of the received power. Moreover, the system performance in terms of the maximum bit-rate and the Bit Error Rate (BER) is investigated in the presence of the Additive White Gaussian Noise (AWGN) channel model. Also, the OFDM waveform is considered herein as an accommodating environment to examine the activity of the intended modulation techniques due to its' efficiency in using the available Bandwidth (BW). Furthermore, a MATLAB simulation is used to implement the promoted system and clarify the advantages and disadvantages of it in comparison with the currently applied 64 QAM.
\end{abstract}

Keywords: Orthogonal Frequency Division Multiplexing (OFDM); higher modulation format; Signal-to-Noise Ratio (SNR); Bit Error Rate (BER); Bandwidth (BW); small cells; macrocells; Additive White Gaussian Noise (AWGN); bit-rate; heterogonous mobile networks.

\section{Introduction}

The increased growth of transmission data over mobile networks considers as an essential driving force for proposing higher modulation formats [1]. Hence, the growing demand for higher bit-rate is requesting a well exploiting for modulation 
formats in the utilised waveform. As such, fostering the required channel capacity of the data-hungry applications can be achieved by developing the applied transmission methods.

Consequently, many research studies have been induced to support a higher channel capacity of future market demand [2]. As such, achieve developed rates of channel capacity at accepted limits of errors get overwhelming interest in terms of R\&D. Thus, recently, great efforts have been made to promote both bit-rate and Bandwidth (BW) efficiency of a transmitted signal by extensively employing higher modulation formats [3].

The diversity of the transmitted signals among users leads to expand the usage of the applied broadband where the mechanism of data transmission is developed from only working with emails to multimedia signals. Hence, nowadays, using smart phones, users are looking for downloading and uploading huge amounts of data rather than the conventional operations [4].

It's worth noting that, picking a suitable modulation format for future wireless networks of mobile depends on the intended performance in terms of the bit-rate, Bit Error Rate (BER) and the coverage of the transmission system. As such, compared to the lower schemes of modulation, the higher modulation formats significantly improve the BW efficiency and maximum bit-rate, whereas, the quality of the transmission service is typically measured by both the BER and the maximum gained rate of bits [5].

In addition, factors like the received power and probability of constellation table noise are major limitations for developers in the modulation format field of wireless communication [6]. Hence, such restrictions can affect the efficiency of spectrum and slow down the speed of transmitting information in an obtainable $\mathrm{BW}[7]$.

The main challenge of the future mobile networks is achieving a higher transmission rate for the mobile signals with lower levels of the BER. Hence, the possibility to employ the modulation process to promote the maximum rates of transmission yet keeping the minimum level of the Signal-to-Noise Ratio (SNR) at the accepted BER limit [8].

Very recently, the digital modulation is considered as one of the most significant issues in the wireless communications world. Hence, utilising an appropriate type of a modulation format for an advanced wireless transmission is a critical due to key limitations of the available transceiver power. As such, the performance in terms of maximum bit-rate and minimum BER of a modulation scheme depends on the efficiency of the BW and power usage [9].

Moreover, the predictably increased data rates for future mobile applications make the currently utilised large macrocells (base station towers) unable to provide efficiently higher bit-rate for the modern telecommunications [10]. Hence, the need for seeking a new approach is quite necessary.

As a result, the potentiality of deploying the small cells technology in the future wireless communication of mobile is highly encouraged [11]. Hence, introducing the small cells as an alternative or supportive technique to the current networks (macrocells) can play a more important role in the modern mobile net- 
works. Thus, the expected topologies of the future networks can be at most as a heterogeneous network, where a mix of large and small cells are employed with variant sizes and power levels [12].

This scalable solution, however, comes up with increasing the computational complexity due to expanding the required number of utilised cells.

The key question of this paper is, can the higher modulation formats in cooperative with the heterogeneous networks be a good solution for a developed performance of future wireless mobile technology?

In this paper, the performance (bit-rate, BER) for the advanced digital modulation schemes, particularly, 128 and 256 QAM is investigated for the first time in diverse scales of wireless mobile networks. Hence, explore how the developed modulation formats can affect the performance of transmission in terms of channel capacity and the BER for the heterogeneous networks that combine both the macrocells and microcells.

Furthermore, the newly introduced modulation formats are examined utilising the most popular transmission air interface, Orthogonal Frequency Division Multiplexing (OFDM). Due to the orthogonality, the current waveform of wireless technology can deliver a better level of transmission service comparing with the old fashion Frequency Division Multiplexing (FDM) [13].

In addition, the channel model, Additive White Gaussian Noise (AWGN), is basically considered herein to explain how this uniform noise can degrade the performance of the transmission system [14].

The rest of the paper is organized as follows: Section 2 discusses theoretically the main concepts of the presented system, highlights the fundamentals beyond it physically and mathematically. Section 3 numerically simulates the performance of the system in terms of the channel capacity and BER. Section 4 summarises the outlines of the paper.

\section{System Model}

In this part, developed formats of modulation/de-modulation process are introduced. The proposed scheme can improve the capacity of the upcoming wireless channel in comparison with the conventional modulation formats. Thus, the advanced kinds of modulation which are higher than the existing 64 QAM are employed to promote the throughput of the transmission for the future mobile communication system $(5 \mathrm{G})$. As such, utilising higher modulation schemes like the 256 QAM, each sub-carrier in the forthcoming networks is eligible to carry 8 bits instead of 6 bits.

In addition, the introduced design is studied in terms of the maximum bitrate and required SNR to explore how the newly proposed modulation formats can impact the performance of transmission in relative to the current wireless system.

As is shown in Fig. 1, the main environment of the transceiver at the physical layer is described. Thus, clarifying the relationship between the developed modulation formats and the other employed parts in the electrical back-to-back wire- 
less transmission system. Hence, the basic phases involving the modulation/demodulation operations of the current wireless mobile waveform (OFDM) are demonstrated herein.

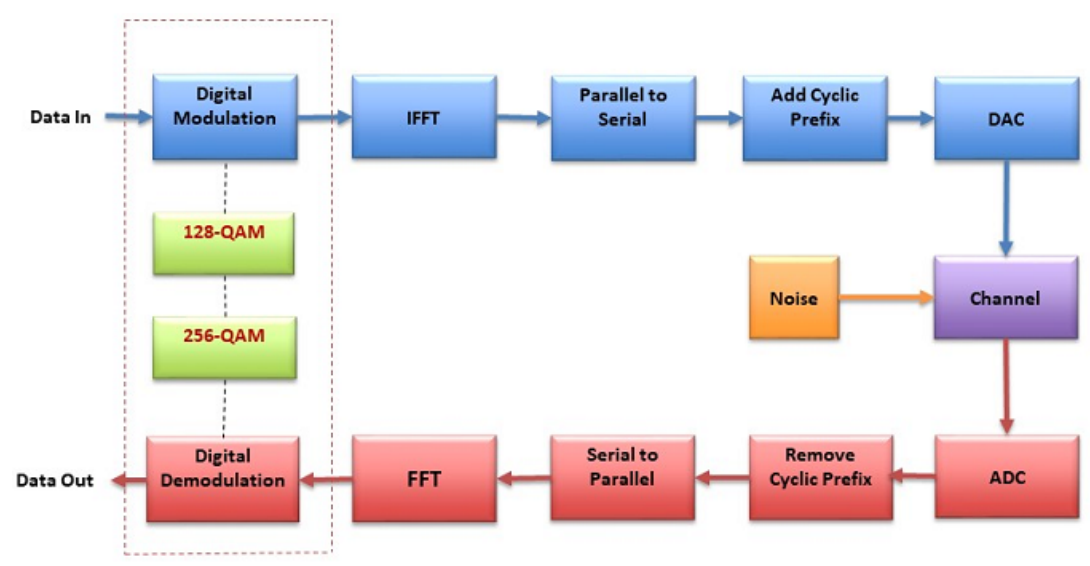

Fig. 1. Proposed design for a future wireless transmission system with higher modulation schemes (128 QAM \& 256 QAM.

Regarding the first level of transmission part (modulation stage), the new design utilises a higher modulation format (128 QAM \& 256 QAM) to convert each token of binary digits $(0,1)$ to its' frequency domain shape.

Since the higher schemes of modulation are used, the supposed size for every token is larger than the currently available length (6 bits). As such, for a stream of binary data, it's much better, in terms of BW efficacy to carry 7 or 8 bits instead of 6 bits as a maximum.

Based on the input stream of digits, the modulation procedure fundamentally comes up with introducing an equivalent set of complex numbers. The new shape of data can work in the frequency domain, hence, specify each complex number to one of the available sub-carriers.

After that, employing the Inverse Fast Fourier Transform (IFFT), the complex number is converted to a new shape in the time domain which is formally called a sample. It's worth noting that, each a fixed group of samples (depending on the number of really offered sub-carriers) termed as a symbol.

In the time domain, where the symbol is prepared for transmission, an important part is attached to the original symbol $(\mathrm{CP})$ providing an extra time protection. Thus, employing an optimal CP, the effect of probably occurred interference among symbols (ISI) is avoided.

Utilising an appropriate $F_{D A C}$, with a recommended high sampling frequency, the digital symbol is transferred to the analog domain. As such, the analog signal is ready now for transmitting by an antenna. 
During the transmission, the broadcasted signal may suffer from variant types of attenuation, which, as a result, can influence the performance of transmission in terms of the BER and maximum gained bit-rate. Herein, the impact of the AWGN channel is considered for exploring the transmitted signal under unwanted conditions.

On the other hand, at the receiver part, an opposite treatment is performed to retrieve the original signal. Thus, the received signal is converted back to digital domain using a similar sampler $F_{A D C}$.

Then after, the supplementary $\mathrm{CP}$ is removed from each symbol, producing the abstract symbol at the time domain. The processed symbol is then changed to the frequency domain using the Fast Fourier Transform (FFT).

The complex numbers, at this domain, are checked to ensure whether their phases and magnitudes were impacted by the channel response or not. The affected complex numbers are addressed utilising the Equalization process which is essentially applied to correct any probable change in both the phase and amplitude.

After the correction operation is completed, a developed de-modulation process is ultimately performed employing higher modulation formats (128 QAM \& 256 QAM), to recover the original stream of binary digits.

As the modulation/de-modulation process represents a key stage of the proposed system, it is quite important to discuss, from a mathematical perspective, some related concepts that can affect directly the transmission operation.

In the transmitter side, particularly, in the modulation process, each token of the binary digits is converted to a complex number which is expressed in Cartesian forms as follow [15]:

$$
C_{k}=I_{k}+j Q_{k}
$$

where $j=\sqrt{-1}$, and $I, Q$ represents the real and imaginary parts for $k^{t h}$ complex number respectively.

In this context, the mathematical relationship between the amplitude (A) and a produced complex number is clarified as follow [16]:

$$
A_{k}=\sqrt{I_{k}^{2}+Q_{k}^{2}}
$$

Regarding the probable shapes number of complex numbers on the constellation map, the following equation achieves this purpose [17]:

$$
Y=2^{X}
$$

where $Y$ represents the total number of probabilities which might be assigned for each sample employing $X$ of input bits.

Despite each spectrum of sub-carrier can coincide with the others, the ability to extract each sub-carrier is achieved over the digital signal processing. Hence, this overlapped property of sub-carriers increases the spectrum efficiency of the current OFDM in comparative with the previous multi-carrier design of 
waveform. Thus, the OFDM technique splits a wireless channel into smaller subcarriers each one is modulated with an amount of data according to the applied modulation format [18].

The improved efficiency of the OFDM spectrum is gained due to applying the orthogonality between adjacent sub-carriers. Consequently, obtain a larger benefit for the same offered BW.

According to Shannon's theorem, the channel capacity represents the maximum achieved bit-rate with a vanished amount of errors as follow [19]:

$$
\text { Capacity }=B W \cdot \log (1+S N R)
$$

In addition, the required SNR to receive the signal is calculated as follow [19]:

$$
S N R=P_{\text {ReceivedSignal }} / P_{\text {Noise }}
$$

In this study, to improve the obtained channel capacity, an extra investigation is performed in the field of BW efficiency than going with expanding the offered BW itself since such expanded for resources requires a highly increased cost.

Besides, the modulation operation is explored in the presence of the AWGN channel. Hence, the received signal $R$ that is composed of the transmitted signal $T$ multiplied by the response of the channel $E$, where $E=1$, is combined with the AWGN which is represented here as $N$, as follows [19]:

$$
R=E T+N
$$

\section{Results and Discussion}

In this part, the promoted wireless transmission system is numerically simulated to demonstrate the advantages and disadvantages of proposing higher modulation formats for the future mobile networks. Hence, the performance in terms of maximum bit-rate and the BER for the OFDM based 128 QAM and 256 QAM is explored.

In addition, the test is set up herein for 15 frequencies of sub-carriers where the developed modulation schemes are considered side by side with the conventional modulation formats. Besides, the optimally utilised parameters of the configured model are undertaken for a wireless electrical back-to-back system. Moreover, to examine the behaviour of wireless channel responses over variant modulation schemes, the MATLAB simulation is equipped.

Since the modulation system is the core of the proposed system, the number of specified bits for each applied sub-carrier is accurately fixed according to the corresponding level of the utilised modulation format. The expanded performance of transmission is achieved under the conditions shown in Table 1.

Regarding the relationship between gained channel capacity and the utilised modulation techniques, as is shown in Fig. 2, the newly proposed modulation 
Table 1. SYSTEM PARAMETERS FOR THE OFDM

\begin{tabular}{|c|c|}
\hline Parameter & Value \\
\hline Modulation Format & BPSK - 256 QAM \\
\hline FFT size & 40 \\
\hline FFT sampling frequency & $20 \mathrm{MHz}$ \\
\hline Number of sub-carriers & 15 \\
\hline Cyclic prefix & 0.25 \\
\hline
\end{tabular}

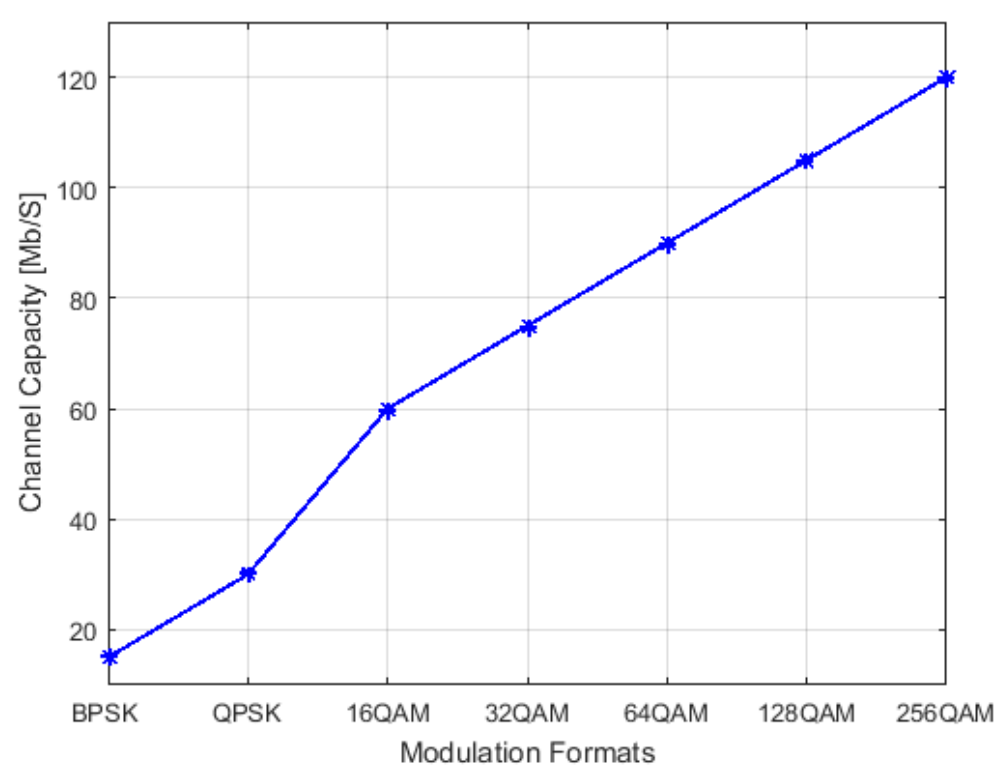

Fig. 2. Maximum achieved bits rates of different modulation formats including the 128 and 256 QAM.

formats, 128, and 256 QAM can increase the transmission bit-rate in compared with the currently applied 64 QAM to about $16 \%$ and $33 \%$ respectively.

Besides, in relative to the $32 \mathrm{QAM}$ and $16 \mathrm{QAM}$, the higher modulation 256 QAM improves the overall channel capacity by around $60 \%$ and $100 \%$ sequentially. In addition, 3 and 7 times enhancement are recorded for both QPSK and BPSK respectively in related to the higher order modulation (256 QAM).

This, however, comes up with raising the level of errors for transmitted samples due to increased interference at the constellation map. Hence, a higher BER is introduced for each employed sub-carrier. Thus, raising a minimum limit of the received power at $10^{-3} \mathrm{BER}$.

The simulated work demonstrates, also, how the BER of the utilised subcarriers are varied for diverse modulation schemes under a similar level of the SNR and over AWGN channel. 
As is seen in Fig. 3, the BERs of the applied frequencies of sub-carriers for the currently employed modulation format (64 QAM) are calculated. The measured BERs are achieved with a good level of the SNR which is equivalent here to $23 \mathrm{~dB}$. Thus, the accepted limit of error is gained due to supplying a suitable standard of received power for the signal modulated with 64 QAM.

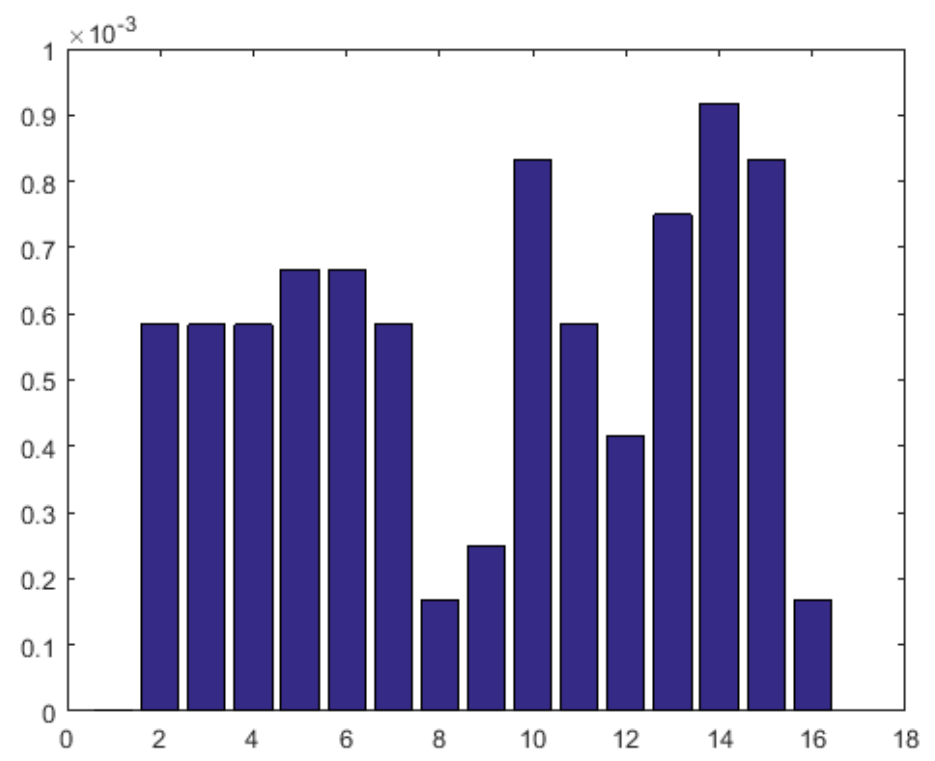

Fig. 3. BER bars of utilised sub-carriers with $64 \mathrm{QAM}$ and $\mathrm{SNR}=23$.

This scenario, nevertheless, is not usually fit for all other modulation formats, particularly, higher order schemes of modulation like 256 QAM.

As is clear in Fig. 4, an extra rise of the BER limit is registered with additional expansion of the modulation format to get the 256 QAM and keep the SNR at the $23 \mathrm{~dB}$. This, essentially, due to decrease the distances between the adjacent samples of the enlarged modulation schemes on the constellation table resulting in un-ability in recognizing the received signal.

To mitigate this issue, the SNR is gradually raised to be more suitable for the 256 QAM. Hence, whenever the received power of the signal is increased the BER is decreased until achieve the accepted criteria of tested modulation scheme.

As is shown in Fig. 5, the overall system performance in terms of the BER and SNR for the most common modulation format with the AWGN channel is presented. Hence, the trade-off relation between the gained BER and offered SNR is digitally processed for variant modulation schemes (QAM and PSK). Thus, compare the transmission performance of conventional modulation techniques 


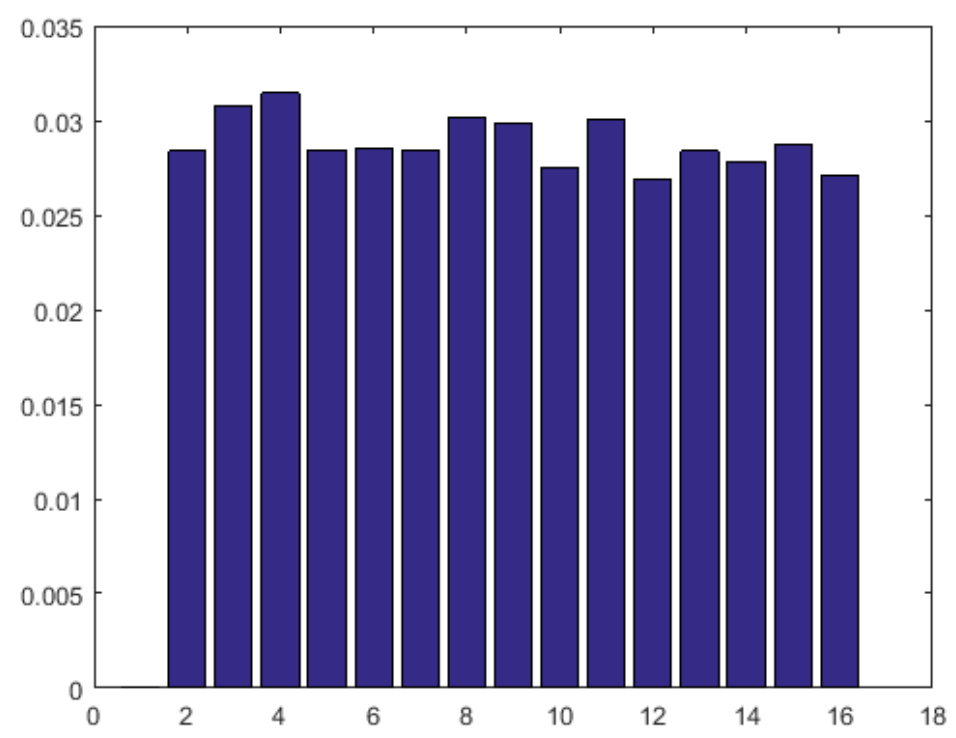

Fig. 4. BER bars of utilised sub-carriers with $256 \mathrm{QAM}, \mathrm{SNR}=23$.

as the BPSK, QPSK,16 QAM, etc, with advanced configurations of modulation (128 and 256 QAM) over the AWGN channel.

It's worth noting that, among these 7 investigated modulation formats, the BPSK can be applied with a lower power requirement, while the 256 QAM requires a higher level of power due to the increment of bits number for each transmitted sample.

As a result, with the future generation of mobile, the higher modulation schemes are very recommended due to the increased need for a higher channel capacity.

Practically speaking, apply higher modulation schemes for an expanded coverage of the signal requires either raising the power of the receiver (higher SNR) or increasing the number of repeater towers (power injection).

Regarding the first option, raise the required power for receiving the signal with a higher modulation scheme can come up with increasing the cost of transmission (more expensive base stations).

As regards the second option, increase the number of repeaters is quite useful if and only if the kind of applied repeater can be offered with better attributes than the current big cells. Thus, the small cell with its' valuable features like simple installation, small size, and low cost can be the best option for this case. Hence, in the case of the higher modulation formats, the small cells repeaters (Femtocells, Picocells, Microcells) can be utilised with close distances to secure a larger transmission coverage. 


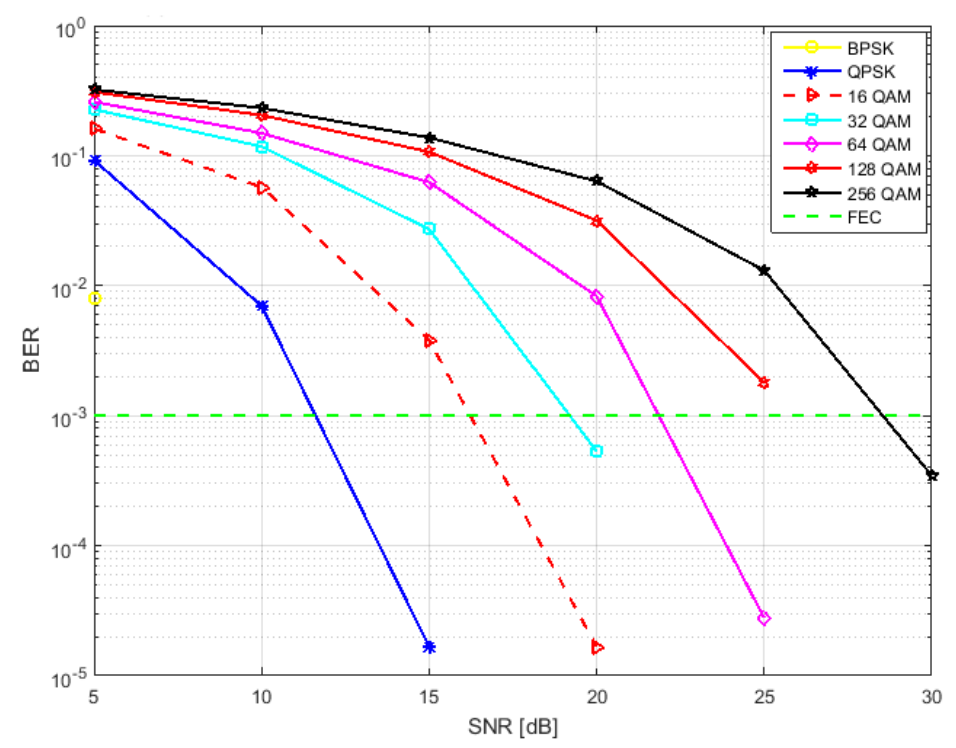

Fig. 5. System performance in terms of BER and SNR for most common modulation formats including both 128 and 256 QAM over AWGN channel.

However, this mix of large and small cells (heterogeneous network) comes up with raising the level of complexity due to the increased number of employed cells.

To explain more about this, it is clear from Fig. 6 that, different levels of the SNR are required to achieve a similar coverage $(D 1)$ in a wireless transmission system with variant shapes of modulation, where $D 1$ represents the supposed distance for transmitting a modulation scheme with a minimum required level of the SNR and accepted limit of the BER $\left(10^{-3}\right)$.

It is noting that, the higher modulation formats suffer from a bigger amount of power consumption due to a raised level of the constellation map noise in comparison to the lower formats. As such, the relatively minor amounts of attenuation might significantly affect the signal with higher modulation order due to close space between samples on the table.

For instants, the minimum limit of received power for the higher order modulation (256 QAM) is larger than the lower order (BPSK) by about four times. Thus, the transmitted signal cannot be received unless this minimum level of power is achieved to ensure accepted limits of the BER.

As is illustrated in Fig. 7, the low modulation schemes can utilise this feature and travel farther distance $(D 2)$ with a good BER in comparison to the high modulation since the obtainable level of power is still supportive of for the low modulation. Hence, $D 2=D 1 * M 1$, where $M 1$ denotes enhancement times of transmission distance due to improved level of the offered SNR with a transmis- 


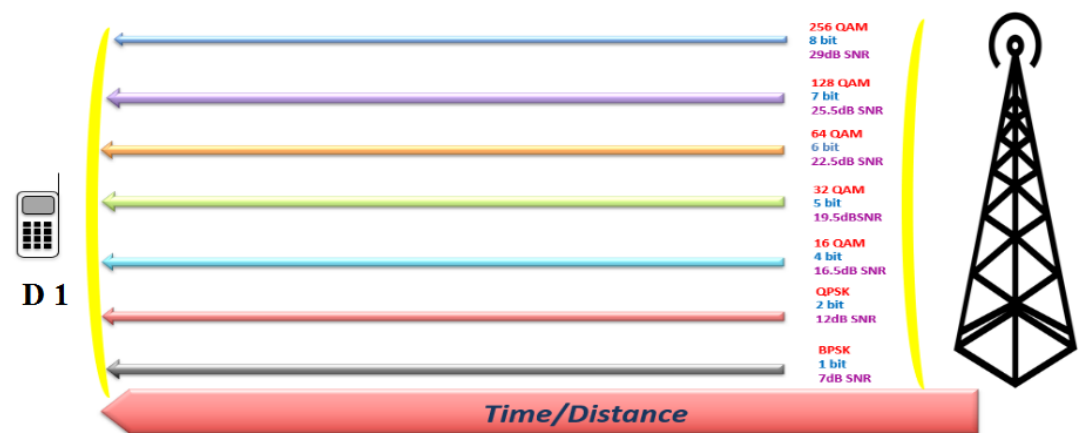

Fig. 6. Different modulation formats with different SNR levels at a wireless transceiver system.

sion system that can basically support high modulation formats. This, however, reduces the bit rate of transmission to a minimum level to keep it constantly at accepted limits of errors.

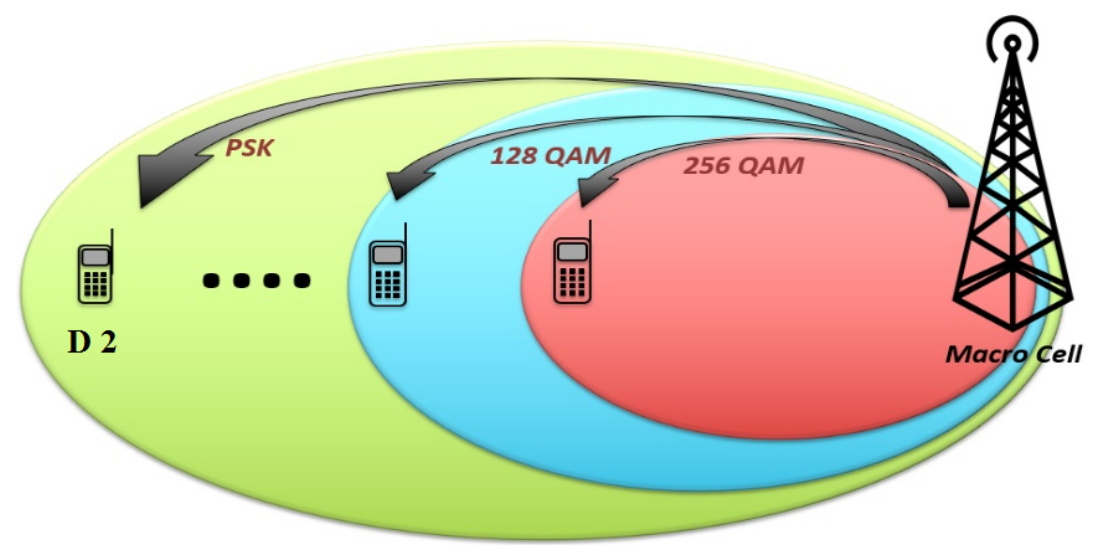

Fig. 7. Macrocell with similar SNR and different modulation formats involving the proposed 128 and 256 QAM.

On the other hand, as is seen in Fig. 8, after proposing the small cells for modern mobile networks, the range of transmission $(D 1)$ is quite expanded to (D2) for the higher modulation scheme (256 QAM) with a lower required limit of the SNR $(29 \mathrm{~dB})$. Hence, $D 2=D 1 * M 2$, where $M 2$, denotes enhancement times of transmission distance due to employing the small cells. Thus, by employing both the high order modulation schemes and the small cells technology, the transmitted signal can move to a longer distance with higher channel capacity and acceptable limits the BER. 


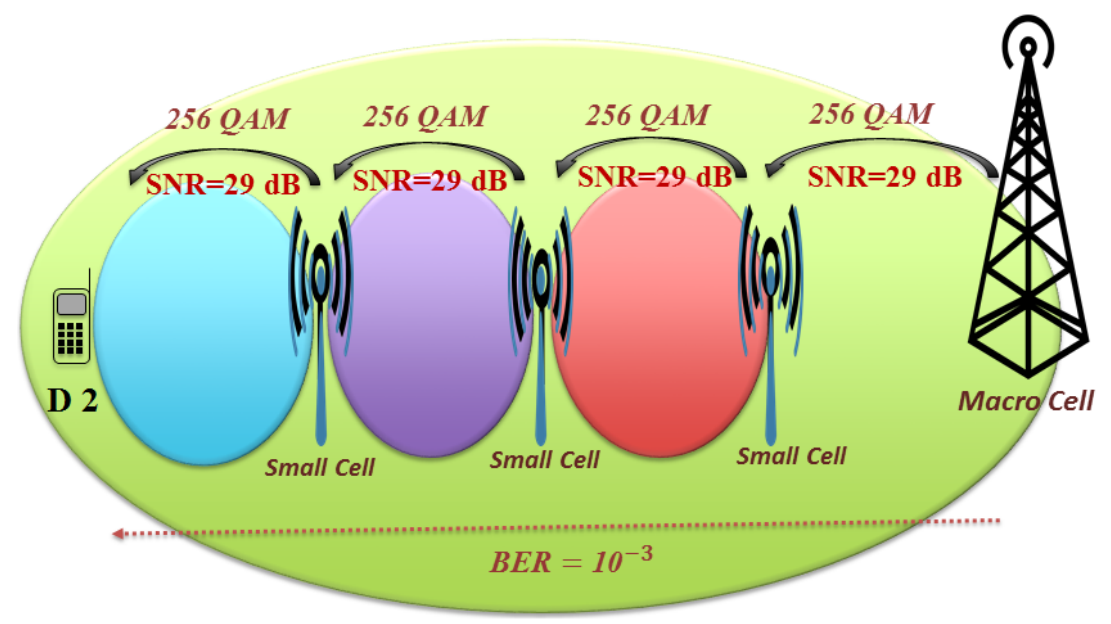

Fig. 8. Utilise both small cells and higher modulation format in one transmission system.

This is essentially occurred, since, the required levels of power are continuously offered by increasing the number of the utilised cells to sustain the wireless signal every time and everywhere depending on the small cells deployment.

\section{Conclusion}

In this study, the higher modulation scheme (256 QAM) with the heterogeneous mobile network is introduced, investigated and evaluated in the PHY of an electrical back-to-back wireless transceiver system. As the higher channel capacity is one of the significant issues for the future generation of mobile, the currently applied macrocells are no longer be accepted with the higher schemes of modulation. The main reason beyond this is that the conventional network (big cell) which tests a higher modulation shape tends to increase the channel capacity with limited coverage of transmission due to the higher power consumption. Hence, according to the obtained results, about 4 times higher SNR ( $29 \mathrm{~dB})$ is required with the 256 QAM to achieve the same short coverage of the BPSK in the macrocell networks. To address this issue, the high order modulation based small cells technique is proposed for the upcoming mobile technology (5G) improving both the BW efficiency and transmission coverage. The findings show that by employing the higher modulation scheme and the microcells together, the wireless signal can travel a long distance with a higher channel capacity and an acceptable BER limit yet keeping the same level of the SNR (29 dB). As such, regulate the trade-off relationship between the maximum achieved bit-rate and the minimum required level of SNR. Thus, the small cell with its' valuable features as simple installation, small size, and low cost can be the best option for the modern mobile networks. Nevertheless, this mix of large and small cells 
(heterogeneous network) comes up with raising the level of the computational complexity due to the increased number of employed cells.

\section{References}

1. Nagarajan, K., Kumar, V. V. \& Sophia, S.: Analysis of OFDM Systems for High Bandwidth Application. 168171 (2017).

2. Kadhum, M. R., Kanakis, T., Al-sherbaz, A. \& Crockett, R.: Digital Chunk Processing with Orthogonal GFDM Doubles Wireless Channel Capacity. 16 (2018). doi:https://doi.org/10.1007/978-3-030-01177-2-53

3. Ndujiuba, C. U., Oni, O. \& Ibhaze, A. E.: Comparative Analysis of Digital Modulation Techniques in LTE 4G Systems. J. Wirel. Netw. Commun. 5, 6066 (2015).

4. Barnela, M.: Digital Modulation Schemes Employed in Wireless Communication: A Literature review. 2, 1521 (2014).

5. Xiao, Y. U. E., Haas, H. \& Member, S.: Index Modulation Techniques for NextGeneration Wireless Networks. 5, 1669316746 (2017).

6. Ghogho, M., McLernon, D., Alameda-Hernandez, E. \& Swami, A.: Channel estimation and symbol detection for block transmission using data-dependent superimposed training. IEEE Signal Process. Lett. 12, 226229 (2005).

7. Kadhum, M. R., Kanakis, T. \& Crockett, R.: Intra-Channel Interference Avoidance with The OGFDM Boosts Channel Capacity of Future Wireless Mobile Communication. in Proceding of computing conference 2019, London (2019).

8. Chandran, I. \& Reddy, K. A.: Comparative analysis of various Channel Estimations under different Modulation Schemes. 1, 832837 (2017).

9. Kadhum, M. R., Kanakis, T. \& Crockett, R.: Dynamic Bit Loading with The OGFDM Waveform Maximises Bit-Rate of Future Mobile Communications. in Proceding of computing conference 2019, London (2019).

10. Jiang, Z. \& Mao, S.: Energy Delay Tradeoff in Cloud Offloading for Multi-Core Mobile Devices. IEEE Access 3, 23062316 (2015).

11. Reed, M. C., Wang, H., Reed, M. C. \& Wang, H.: Small cell deployments: system scenarios, performance, and analysis. (2018).

12. 3Gpp. The Mobile Broadband Standard. 9, 16 (2014).

13. Jin, W. et al.: Improved Performance Robustness of DSP-Enabled Flexible ROADMs Free from Optical Filters and O-E-O Conversions. J. Opt. Commun. Netw. 8, 521 (2016).

14. Reddy, K. A.: Evaluation of BER for AWGN, Rayleigh Fading Channels under M-QAM Modulation Scheme. 30813086 (2016).

15. Ingle, V. K. \& Proakis, J. G.: Digital Signal Processing Using MATLAB. (2012).

16. Tao, L. et al.: Experimental demonstration of $10 \mathrm{~Gb} / \mathrm{s}$ multi-level carrier-less amplitude and phase modulation for short range optical communication systems. Opt. Express 21, 6459 (2013).

17. Alexander, E. \& Poularikas, D.: The Handbook of Formulas and Tables for Signal Processing. (1999).

18. Stern, S. \& Fischer, R. F. H.: OFDM vs . Single-Carrier Modulation: A New View on the PAR Behavior. 112119 (2014).

19. Im, G. H. et al.: $51.84 \mathrm{Mb} / \mathrm{s}$ 16-CAP ATM LAN Standard. IEEE J. Sel. Areas Commun. 13, 620632 (1995). 